\title{
Detection of splenic microabscesses with ultrasound as a marker for extrapulmonary tuberculosis in patients with HIV: A systematic review
}

\author{
J M Schafer, ${ }^{1}$ MD; J Welwarth, ${ }^{2} \mathrm{DO} ;$ V Novack, ${ }^{3} \mathrm{MD}$, PhD; D S Balk, ${ }^{1} \mathrm{MD}$; T Beals, ${ }^{1} \mathrm{MD} ; \mathrm{L}$ Naraghi, ${ }^{4} \mathrm{MD}$; E K Khattab, ${ }^{5} \mathrm{MD}, \mathrm{MPH}$; \\ B Hoffmann, ${ }^{1} \mathrm{MD}, \mathrm{PhD}, \mathrm{RDMS}$ \\ ${ }^{1}$ Department of Emergency Medicine, Beth Israel Deaconess Medical Center, Harvard Medical School, Boston, Mass, USA \\ ${ }^{2}$ Department of Emergency Medicine, Wayne State University School of Medicine, Detroit, Mich., USA \\ ${ }^{3}$ Soroka University Medical Center, Beer Sheba, Israel \\ ${ }^{4}$ Maimonides Medical Center, Brooklyn, NY, USA \\ ${ }^{5}$ Department of Emergency Medicine, King Khalid University Hospital, King Saud University, Riyadh, Saudi Arabia
}

Corresponding author: J M Schafer (jmschafe@bidmc.harvard.edu)

\begin{abstract}
Background. In 2015, 1.2 million new cases of tuberculosis (TB) were diagnosed in patients with HIV. Diagnostic limitations and resource shortages in endemic areas can delay diagnosis and treatment, particularly with extrapulmonary TB (EPTB). Research suggests that ultrasound can identify splenic microabscesses caused by EPTB, but data are limited on the frequency of this finding in patients with culture-proven EPTB.

Objectives. To estimate the frequency of splenic EPTB microabscesses detected with ultrasound in patients with HIV and TB co-infection. Methods. Studies published in six major databases as of November 2017 were systematically reviewed based on the PRISMA guidelines. Cohen's kappa test was used to determine inter-rater agreement. Articles included for data abstraction passed the Quality Assessment of Diagnostic Accuracy Studies (QUADAS-2) evaluation. Freeman-Tukey transformation was used to calculate weighted proportions. Heterogeneity was evaluated by Forest plot and $I^{2}$ calculation.

Results. After abstract screening, article review and QUADAS-2 evaluation, five studies were selected for data extraction. A total of 774 patients in these studies were infected with HIV. Splenic lesions were seen with ultrasound in $21.0 \%$ of patients with HIV (95\% confidence interval (CI) 10.6 - 33.8). TB diagnosed by culture, biopsy, smear, or molecular methods was found to be the cause of $88.3 \%$ (95\% CI 72.3 - 97.9) of splenic microabscesses seen on ultrasound in patients with HIV.

Conclusions. Ultrasound evaluation of the spleen in patients with HIV and symptoms suggestive of TB in endemic regions is a viable diagnostic adjunct. Ultrasound detection of splenic microabscesses in HIV patients is probably sufficient indication to initiate TB treatment prior to obtaining culture data. Strong conclusions cannot be drawn owing to the high heterogeneity of this small number of studies.
\end{abstract}

S Afr Med J 2019;109(8):570-576. DOI:10.7196/SAMJ.2019.v109i8.13783

The twin epidemics of HIV and tuberculosis (TB) have resulted in a surge in HIV-TB co-infection. In 2015, 1.2 million new cases of $\mathrm{TB}$ were diagnosed in patients living with HIV worldwide, with 400000 deaths. ${ }^{[1,2]}$ The burden of this epidemic is most prominent in the developing world, where resources for diagnosis and treatment are limited, which further hampers containment of the epidemic. ${ }^{[1-3]}$ Typically, $10-15 \%$ of patients with TB develop the infection in sites other than the lungs, i.e. extrapulmonary TB (EPTB).$^{[4-8]}$ Among people living with HIV, EPTB can occur in $>50 \%$ of cases. ${ }^{[4,5,7-9]}$ The diagnosis of TB is usually made by sputum smear, culture, biopsy, or molecular detection methods. Diagnosing EPTB in patients with HIV, however, is challenging. In the absence of active pulmonary TB and in the presence of HIV, sputum smears or cultures to diagnose EPTB are unreliable and can be positive in as few as $3 \%$ of patients. ${ }^{[6,10,11]}$ Likewise, chest radiographs are not helpful in EPTB and are negative in up to $27 \%$ of cases. ${ }^{[12]}$ In resource-limited settings, the diagnosis of EPTB is often made on the basis of clinical findings alone, which can lead to a delay in diagnosis and increased mortality. While molecular methods of TB detection are improving and can offer rapid turnaround of results, they are not yet widely available. ${ }^{[13-15]}$
Recently ultrasound, particularly point-of-care ultrasound (POCUS), has emerged as a useful modality to diagnose advanced EPTB. ${ }^{[16]}$ Its use has increased in resource-limited settings, as it is a cheap, portable and repeatable diagnostic modality that offers rapid data return. ${ }^{[17-19]}$ Typical EPTB ultrasound findings are free abdominal fluid, pericardial effusion, periaortic lymph nodes and splenic microabscesses. ${ }^{[20-26]}$ It is known that both HIV and TB can cause free intra-abdominal or pericardial fluid and lymphadenopathy independently, but splenic microabscesses are believed to be very specific for EPTB. ${ }^{[27,28]}$

The focused assessment with sonography for HIV-associated TB (FASH) protocol was developed to enable treating doctors to identify EPTB rapidly in patients with HIV in endemic areas. ${ }^{[16]}$ The developers of this protocol argued that the presence of positive FASH findings in the right setting indicates disseminated EPTB that should prompt treatment without waiting for additional diagnostic data. ${ }^{[16,21]}$ However, much of the published research supporting the FASH protocol is based on ultrasound findings or clinical progression alone rather than culture data. ${ }^{[10,21,27-30]}$ There is a paucity of systematic data on the frequency and specificity of splenic microabscesses seen on ultrasound in patients with culture-proven EPTB, and 
other organisms aside from the tubercle bacterium can cause disseminated splenic abscess development. ${ }^{[31,32]}$

\section{Objectives}

The objective of this systematic review was to determine the sonographic frequency of TB splenic microabscesses in patients with HIV and confirmed TB co-infection.

\section{Methods \\ Search strategy, selection criteria and assessment of quality}

A systematic review was conducted of all published literature in PubMed, Embase, Web of Science, Cumulative Index to Nursing and Allied Health Literature (CINAHL), Latin American and Caribbean Health Sciences Literature (LILACS) and African Index Medicus (AIM) publications prior to 28 November 2017. Updates were conducted in November 2016 and November 2017. Keywords and search terms were generated for $\mathrm{TB}$ and ultrasound with the help of a research librarian (Appendix 1). Search strategy and methods were based on the PRISMA (Preferred Reporting Items for Systematic Reviews and Meta-analyses) checklist. ${ }^{[33]}$ The resulting database of articles was further refined with addition of HIV keywords. The inclusion criteria were not limited on the basis of language, geographical region, year, or study setting income stratification. Two independent reviewers screened abstracts for exclusion criteria, which were as follows: non-human studies, case reports, case series with three or fewer subjects, or studies relying on invasive ultrasound modalities (trans-oesophageal or endoscopic) alone as the basis for imaging evaluation. A third reviewer was used to gain consensus when conflict arose. Abstracts were included if all study participants had known HIV status and underwent noninvasive abdominal ultrasonography, and TB infection status was determined on the basis of confirmatory data such as culture, biopsy, smear, or molecular detection methods. Articles included for full review were assessed for quality with the Revised Quality Assessment of Diagnostic Accuracy Studies (QUADAS-2) tool. ${ }^{[34]}$ With this tool, articles meeting the inclusion criteria were screened for possible selection, performance and reporting bias. Articles that passed this screening step were included for final data extraction and statistical analysis.

\section{Data abstraction}

Data extraction was standardised to include geographical location of the study, study design, characteristics of the population such as HIV and TB status, and method of determining TB status. Data regarding presence or absence of splenic lesions on ultrasound found with the use of either low-frequency or high-frequency ultrasound transducers were also abstracted. Authors of the selected studies were contacted as needed to clarify any missing data such as HIV status or ultrasound transducer used for evaluation of splenic lesions.

\section{Statistical analysis}

During screening, inter-rater agreement was assessed using Cohen's kappa test. Data from studies that passed the QUADAS-2 evaluation were pooled to calculate three parameters: ( $i$ ) the rate of $\mathrm{TB}$ splenic microabscesses in patients with HIV-TB co-infection; (ii) the rate of splenic lesions in all patients with HIV; and (iii) the rate of $\mathrm{TB}$ microabscesses out of all splenic lesions in patients with HIV. The FreemanTukey transformation (arcsine square root transformation) was used to calculate the weighted summary proportion for each of these parameters under the fixed and random-effects model. ${ }^{[35,36]}$ Heterogeneity was assessed by graphic examination of Forest plots and by calculating $I^{2}$ and Cochrane's $Q{ }^{[37]}$ Publication bias was assessed by Egger's regression intercept. ${ }^{[38]}$ We used MedCalc Statistical Software version 14.8.1 (MedCalc Software, Belgium; http://www.medcalc.org,
2014) and Comprehensive Meta-Analysis version 2.2.064 (Biostat, USA). A two-tailed $p$-value $<0.05$ was considered statistically significant.

\section{Role of the funding source}

There was no funding source for this study. The corresponding author had full access to the data in the study and final responsibility for the decision to submit for publication.

\section{Results}

Search and screening results are listed in Fig. 1. The initial search returned 5545 unique articles for TB and ultrasound. The addition of HIV keywords a priori to this database further refined the results to 993 abstracts. Two reviewers screened these abstracts, returning 54 articles pertaining to splenic lesions on ultrasound in patients with HIV and TB co-infection. Reviewers had a kappa coefficient of 0.9 (95\% confidence interval (CI) 0.7 - 1) for interrater agreement. After the QUADAS-2 tool was applied, five studies were selected for data extraction. The other 49 articles were excluded for the following reasons: 7 were case control studies; 22 based the diagnosis of EPTB on clinical or empirical parameters rather than strictly culture, biopsy, smear, or molecular data, or there was insufficient information on methods of diagnosing TB; 5 studies had mixed use of either computed

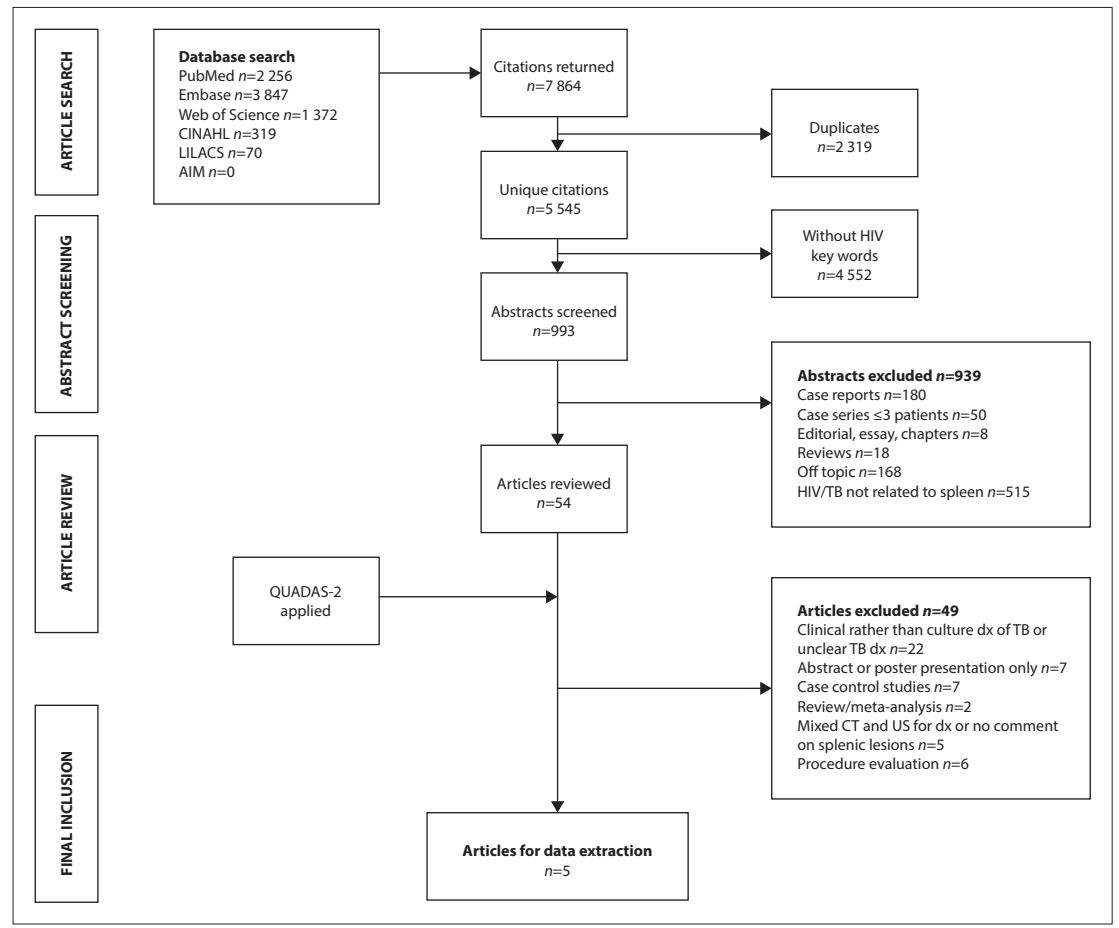

Fig. 1. Systematic review search and screening results. (CINAHL $=$ Cumulative Index to Nursing and Allied Health Literature; LILACS = Latin American and Caribbean Health Sciences Literature; AIM = African Index Medicus; TB = tuberculosis; QUADAS-2 = Quality Assessment of Diagnostic Accuracy Studies; $d x=$ diagnosis; $C T=$ computed tomography; US = ultrasound.) 
tomography or ultrasound as the imaging modalities, or did not comment on splenic ultrasound findings; 2 were either reviews or meta-analyses; 6 evaluated procedures; and 7 were only available in limited abstract or poster presentation form. Table 1 lists the five selected articles and the key characteristics of each.

\section{Pooled results}

The five studies included a total of 840 patients who had an abdominal ultrasound scan. Of these patients, 774 had HIV infection. TB co-infection was confirmed in 337 of patients with HIV. Ultrasound detected hypoechoic splenic lesions in 151 of the 840 patients. Splenic microabscesses found on ultrasound were confirmed to be EPTB in 137 of these patients.

Forest plots, $I^{2}$ and Cochrane's Q-tests were used to evaluate heterogeneity of these studies. The Forest plots with associated $I^{2}$ and Cochrane's Q-values of pooled data from the five selected studies are listed in Figs 2 - 4. There was high heterogeneity among the studies, so weighted summary proportion was calculated using FreemanTukey transformation (arcsine square root transformation) under a random-effects model.

The proportion of splenic microabscesses in patients with HIV and TB co-infection is presented in Fig. 2. There was high heterogeneity, with Cochrane's $Q$ at 66.9 $(p<0.0001)$ and $I^{2} 94.0 \%$ (95\% CI 88.9 - 96.8), so a random-effects model was used. Of the 337 patients with HIV-TB co-infection, $62.5 \%$ had splenic microabscesses with culture-proven TB (95\% CI 38.1 - 83.9). Egger's regression intercept to evaluate publication bias was 2.62 (95\% CI -0.3 - 5.5; $p=0.064$ ), indicating that no publication bias was found.

The proportion of splenic lesions on ultrasound in all patients with HIV is shown in Fig. 3. Cochrane's $Q$ was $63.2(p<0.0001)$ and $I^{2}$ was $93.7 \%$ (95\% CI 88.1 - 96.6), indicating high heterogeneity, so a randomeffects model was used. Of 774 patients with HIV, splenic lesions were seen in 21.0\% (95\% CI 10.6 - 33.8). Egger's regression to assess publication bias was -7.3 (95\% CI -30.716.2; $p=0.4$ ), indicating that no publication bias was found.

The proportion of TB microabscesses out of all splenic lesions in patients with HIV is shown in Fig. 4. Cochrane's $Q$ was 24.6 $(p<0.0001)$ and $I^{2}$ was $83.8 \%$ (95\% CI 63.3 92.8), indicating high heterogeneity, so a random-effects model was used. Of patients with HIV and splenic microabscesses on ultrasound, $88.3 \%$ had TB diagnosed by culture, biopsy, smear or molecular methods (95\% CI 72.3 - 97.9). Egger's regression intercept was 4.6 (95\% CI $-1.7-11.0 ; p=0.10)$, indicating that no publication bias was found.

\section{Results of individual studies}

Murray et al. ${ }^{[39]}$ (1995) compared rates of splenic microabscess detection between a 3.5 $\mathrm{MHz}$ sector transducer and a $5 \mathrm{MHz}$ linear transducer in patients with HIV. This study was done in the USA on a prospective convenience sample of patients with known HIV status presenting for investigation of abdominal pain between June 1993 and October 1994. Of 102 patients who underwent abdominal ultrasonography, 13 were found to have splenic lesions. Use of a low-frequency transducer was able to identify splenic lesions in 3 patients, while an additional 10 patients were recategorised as having lesions after evaluation with a high-frequency linear probe. Seven of these patients had a TB diagnosis based on culture, biopsy or autopsy, 3 had Mycobacterium avium-intracellulare infection, 1 had Kaposi's sarcoma, and 1 had Pneumocystis carinii infection. The remaining patient was presumed to have disseminated candidiasis based on ultrasound appearance of the splenic lesion and improvement with antifungal medication.

Porcel-Martin et al. ${ }^{[40]}$ (1998) published a retrospective review of patients with HIV who underwent abdominal ultrasonography between January 1987 and December 1995 at one hospital in Spain. The objective of their research was to describe splenic ultrasound findings in this patient population. They used $3.5 \mathrm{MHz}$ sector and linear transducers to evaluate the spleen. Of 22 patients found to have focal splenic lesions, 19 had cultureor biopsy-proven ЕРТВ. One patient had a splenic infarct from Staphylococcus aureus bacterial endocarditis, and in 2 patients the final diagnosis was unknown because of lack of follow-up.

Mendez et al. ${ }^{[41]}$ (1995) evaluated 77 patients in Argentina who presented with known HIV and fever. This was a prospective cohort study to evaluate the usefulness of ultrasound in detecting abdominal TB in HIV-positive patients. They used $3.5 \mathrm{MHz}$ sector and linear transducers. Of 74 patients with culture- or biopsy-proven TB, 29 had splenic microabscesses with confirmed TB.

More recently, in 2011, Patel et al. ${ }^{[42]}$ published a prospective cohort study of ultrasound findings in patients with suspected EPTB admitted to a district-level hospital in South Africa from August 2004 to October 2004. Ultrasound of the spleen

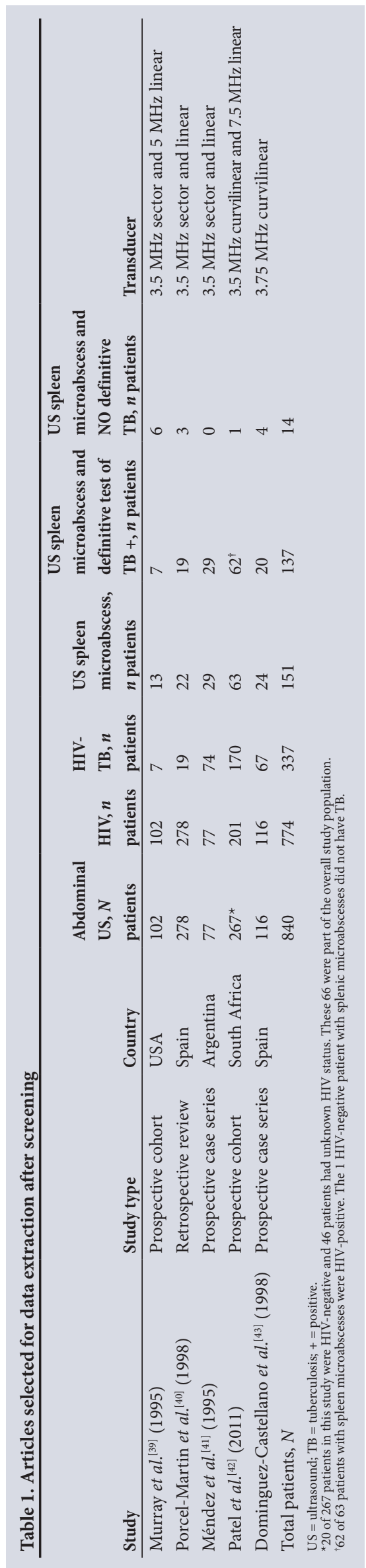




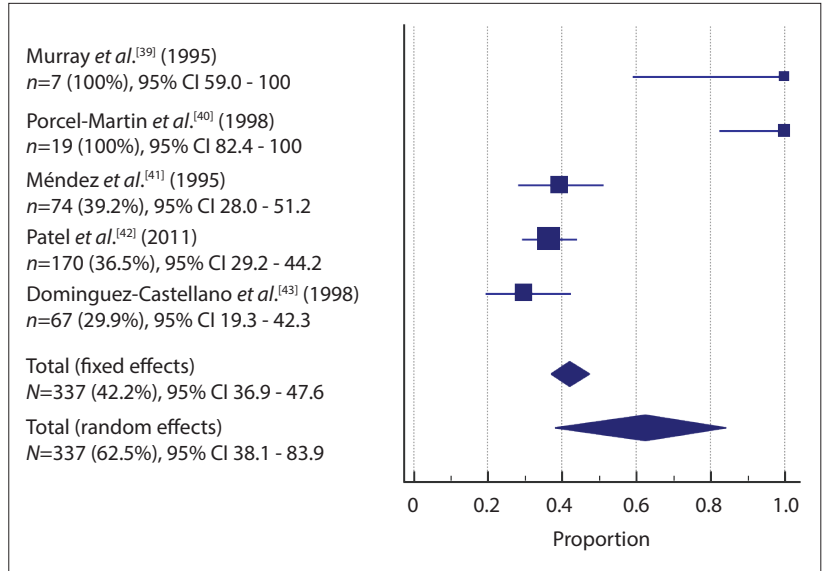

Fig. 2. Forest plot of selected articles: rate of $T B$ splenic microabscesses in patients with HIV-TB co-infection. $(T B=$ tuberculosis; $C I=$ confidence interval.)

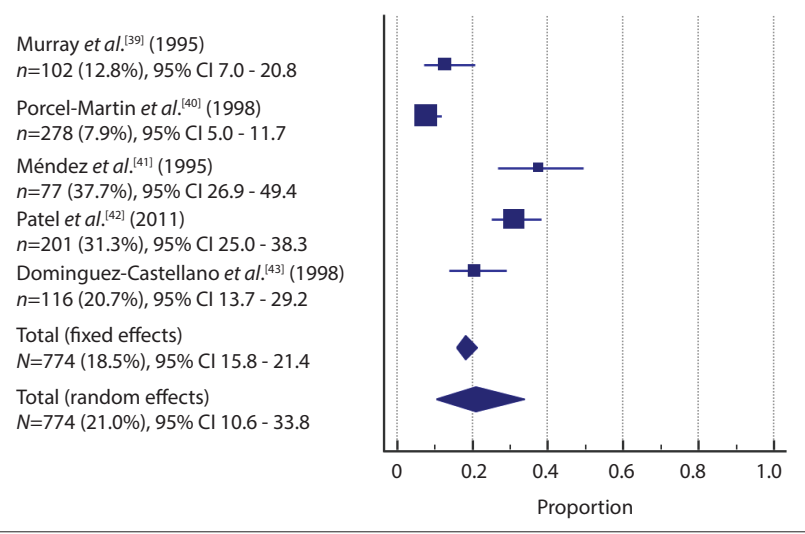

Fig. 3. Forest plot of selected articles: rate of splenic lesions in patients with HIV. (CI = confidence interval. $)$

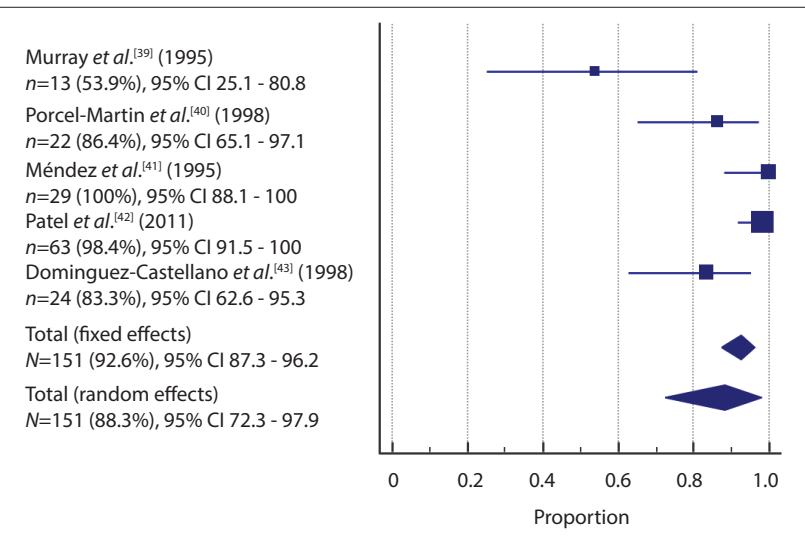

Fig. 4. Forest plot of selected articles: rate of TB splenic microabscesses $v$. all splenic lesions in patients with HIV. (TB = tuberculosis; $C I=$ confidence interval.)

was performed using a $3.5 \mathrm{MHz}$ curvilinear transducer and a 7.5 MHz linear transducer. Of 267 patients who met the inclusion criteria and had adequate data to confirm a diagnosis of TB, 170 had TB confirmed by either culture or smear. Of the 267 patients in this study, 66 were either HIV-negative or of unknown HIV status, while the remaining 201 were HIV-positive. Of 63 patients found to have splenic microabscesses, the majority $(n=62)$ were HIV-positive with a final diagnosis of EPTB. The single HIV-negative patient in this group with splenic lesions did not have TB. The authors did not specify what the final diagnosis was in this case.

Dominguez-Castellano et al. ${ }^{[43]}$ (1998) conducted a prospective cohort study of patients admitted to one hospital in Spain from April 1994 to January 1997. The objective of this study was to evaluate abdominal ultrasound as a diagnostic tool to identify EPTB in patients with HIV. They used a $3.75 \mathrm{MHz}$ curvilinear transducer to scan for splenic lesions, abdominal lymphadenopathy or liver lesions. Of 116 patients with HIV and fever evaluated with abdominal ultrasound, 35 had a combination of abdominal ultrasound findings and 25 of these had a final diagnosis of EPTB; 24 patients had splenic microabscesses on ultrasound. Of these microabscesses, 20 were biopsy- or culture-proven TB, while 1 was caused by toxoplasmosis and 1 by $M$. avium-intracellulare. In 2 cases there was no definitive diagnosis. The authors noted that one patient with ultrasound findings had a presumptive diagnosis of TB based on clinical improvement, but they did not state what specific ultrasound finding this case was associated with.

\section{Discussion}

Five studies that used abdominal ultrasound in the diagnostic algorithm when evaluating for EPTB were reviewed. Each of these studies were selected because the diagnosis of TB was confirmed by culture, biopsy, smear, or molecular methods. HIV status was known in a majority of patients included in this review (794/840 patients, 774 HIV-positive and 20 HIV-negative). Splenic microabscesses were present in $21.0 \%$ of all patients with HIV infection. Of patients with HIV and TB co-infection, $62.5 \%$ had splenic microabscesses. Furthermore, in patients with HIV, these splenic microabscesses were caused by culture-proven EPTB in $88.3 \%$ of cases. The above data indicate that splenic microabscesses were not a frequent finding, but when present were usually caused by EPTB. Therefore, in HIVpositive patients with unknown TB status but symptoms suggestive of TB, splenic microabscesses detected on ultrasound are probably caused by EPTB, and this possibility may be sufficient indication to initiate TB treatment while awaiting culture data, particularly in a setting with a high prevalence of HIV and TB and limited resources.

A strength of this review is that we focused on diagnostic modalities likely to be available to the front-line provider. The review focused primarily on transabdominal ultrasound and excluded invasive ultrasound modalities, as these do not easily translate to POCUS applications. Invasive modalities would probably not be available to the front-line clinician practising in an HIV-TB-endemic area with limited resources. It was with this subset of clinicians in mind that we chose to focus on splenic microabscesses as an ultrasound finding associated with EPTB.

There are other ultrasound findings commonly associated with EPTB that we could have investigated, but these findings present their own challenges. Abdominal lymphadenopathy is a common finding with EPTB in patients with HIV; however, abdominal lymph nodes can be difficult to identify, even for experienced sonographers. ${ }^{[31,44]}$ Ascities is associated with TB but also with a wide variety of other conditions. ${ }^{[45]}$ We therefore chose to focus on splenic microabscesses, as they are relatively easy for a clinician with limited ultrasound experience to identify. ${ }^{[46,47]}$ Given that POCUS is cheap and portable, and provides data rapidly to the treating clinician, ultrasound evaluation of the spleen in patients with HIV and symptoms suggestive of TB is a beneficial addition to diagnostic and treatment algorithms in resource-limited settings.

During the screening process, we found 22 articles that discussed the presence of splenic microabscesses in patients with HIV and TB co-infection. ${ }^{[12,21,24,27,28,30,48-63]}$ These articles were excluded because 
TB was diagnosed either entirely or partially on the basis of clinical or ultrasound features rather than strictly by culture, biopsy, or molecular methods. Smear-negative TB and EPTB can be difficult to diagnose, particularly in patients with $\mathrm{HIV}^{[6,10-12]}$ Criteria for diagnosis are often based on clinical signs or symptoms as well as improvement in symptoms after initiation of TB treatment, because EPTB or smear-negative TB is so difficult to diagnose. ${ }^{[10,29]}$ The excluded articles followed World Health Organization guidelines for diagnosing EPTB, and were well structured; however, we were looking for research that indicated a definitive culture- or biopsyproven diagnosis of TB, as patients with HIV are also susceptible to other opportunistic infections or malignancy that can present in a similar way. Looking only for articles with a definitive TB diagnosis introduces potential bias into our screening process. However, taken together the excluded articles indicate that splenic microabscesses appear to be a very specific finding associated with EPTB in HIVpositive patients, particularly in endemic areas. Our findings add support to this observation.

\section{Study limitations}

As with any systematic review, publication bias is a problem. Using the methods we have described, we searched six major databases for published articles. However, published articles do not encompass the entire breadth of research on this topic, and we did not include unpublished research that might have been relevant. Additionally, through the screening and selection process we identified very few articles that used culture or biopsy data as the basis of definitive EPTB diagnosis. We attempted to evaluate for potential publication bias using Egger's regression intercept, but this is of limited usefulness with such a small number of articles. ${ }^{[38]}$ Publication bias as assessed with our method was low; however, considering the research and publication barriers faced by clinicians and researchers in low- and middle-income countries, it is possible that there are unpublished data on this topic. ${ }^{[64-66]}$ Our assessment of publication bias must therefore be interpreted with these limitations in mind.

We noted high heterogeneity in the five selected articles. Differences in study objectives, changes in geographical and temporal distribution of TB and HIV in relation to the growing HIV epidemic, and some of the diagnostic methods used contribute to this heterogeneity. We adjusted for this problem by using a random-effects model to calculate probability. Even so, the estimates presented are not robust owing to the limited number of heterogeneous studies that passed the screening process, and strong conclusions cannot be drawn based on these data.

One of the differences noted between articles was ultrasound transducer selection. Four of the five articles reported using two types of transducers to evaluate the spleen, either a sector or curvilinear transducer and a linear transducer. All five used a low-frequency transducer of 3.5 or $3.75 \mathrm{MHz}$, which is a standard low-frequency transducer for abdominal ultrasound scans. A linear transducer typically offers higher-frequency settings and therefore better resolution. Two investigators indicated that they used an additional high-frequency linear transducer of either 5 or $7.5 \mathrm{MHz}$ for splenic tissue. ${ }^{[39,42]}$ Two other studies indicated that a high-frequency linear transducer was used, but did not indicate the frequency. ${ }^{[40,41]}$ Higher-frequency ultrasound transducers are not routinely used for abdominal examinations, but the rationale behind their use in this context is that they can show more detailed parenchymal images and may be more sensitive for detecting splenic microabscesses.

Murray et al. ${ }^{[39]}$ identified splenic lesions with the $3.5 \mathrm{MHz}$ frequency transducer in 3 out of 13 patients, but found 4 additional patients when the $7.5 \mathrm{MHz}$ transducer was used. Three other studies used a high-frequency linear transducer in addition to the standard
3.5 MHz transducer, but did not specify whether additional patients were identified by using the high-frequency transducer. ${ }^{[40-42]}$ One of the studies only used a low-frequency transducer. ${ }^{[43]}$ The differences in transducer frequency selection among these studies could have the potential to falsely underestimate the frequency of splenic lesions. However, future research evaluating POCUS for this approach should include a low- and high-frequency transducer approach to potentially increase the sensitivity of splenic microabscess detection.

We did not exclude articles on the basis of geographical region or time in this search. Both high- and low- to middle-income countries are represented and an almost 20-year span is covered, starting in the mid-1990s. The shifting demographics of the HIV epidemic are likely to have changed the applicability of these findings in high-income countries, where the incidences of HIV and TB are substantially lower than in low- or middle-income countries.

\section{Conclusions}

The twin epidemics of HIV and TB are fuelling a rise in cases of EPTB, particularly in the developing world. Ultrasound is a cheap and portable diagnostic tool that can offer a partial solution to the difficulty of diagnosing EPTB in a resource-limited setting. While this review focused on sonographic detection of splenic microabscesses, there are other ultrasound findings associated with EPTB in patients with HIV, such as pericardial effusion or periaortic lymph node enlargement. While many of these findings have not been validated, additional meta-analysis of the current literature on the frequency of these ultrasound findings may help strengthen available data for diagnosing EPTB in at-risk patients using POCUS. In our review, we found that splenic microabscesses in patients with HIV are frequently caused by EPTB. This ultrasound finding in patients with HIV living in TB-endemic areas should prompt the clinician to initiate anti-TB treatment, prior to any confirmatory laboratory data. However, strong conclusions cannot be drawn because of the high heterogeneity of the limited number of studies that passed the search and screening process.

\section{Declaration. None.}

Acknowledgements. Thanks to Paul Bain, PhD, MLIS, reference and education librarian at Countway Library of Medicine at Harvard Medical School, for assistance in generating search terms for each database. Author contributions. JMS and BH conceived the protocol. JMS did the literature search. JMS, BH and JW did the abstract screening and article review. JMS and BH did the quality assessment. JMS did the data extraction and contributed to the figures and tables. VN contributed to data analysis, created the figures and edited the manuscript. JMS prepared the first draft of the manuscript, and all authors were involved in data interpretation. All authors (JMS, JW, VN, DSB, TB, LN, EKK and BH) critically revised al drafts of the manuscript and approved the final version.

Funding. None.

Conflicts of interest. None.

1. World Health Organization. Global Tuberculosis Report 2016. Geneva: WHO, 2016 http://www searo. who.int/tb/documents/global-tuberculosis-report-2016/en/ (accessed 20 January 2017).

Corbett EL, Watt CJ, Walker N, et al. The growing burden of tuberculosis: Global trends and Corbett EL, Watt CJ, Walker N, et al. The growing burden of tuberculosis: Global trends and
interactions with the HIV epidemic. Arch Intern Med 2003;163(9):1009-1021. https://doi.org/10.1001/ interactions with the
archinte.163.9.1009

3. Perkins MD, Cunningham J. Facing the crisis: Improving the diagnosis of tuberculosis in the HIV era. J Infect Dis 2007;196(Suppl 1):S15-S27. https://doi.org/10.1086/518656

4. Ade S, Harries AD, Trebucq A, et al. National profile and treatment outcomes of patients with extrapulmonary tuberculosis in Benin. PloS One 2014;22;9(4):e95603. https://doi.org/10.1371/journal. pone. 0095603

5. Berg S, Schelling E, Hailu E, et al. Investigation of the high rates of extrapulmonary tuberculosi in Ethiopia reveals no single driving factor and minimal evidence for zoonotic transmission of Mycobacterium bovis infection. BMC Infect Dis 2015;15:112. https://doi.org/10.1186/s12879-015$0846-7$ 
6. Kwanjana IH, Harries AD, Hargreaves NJ, van Gorkom J, Ringdal T, Salaniponi FM. Sputum-smear examination in patients with extrapulmonary tuberculosis in Malawi. Trans R Soc Trop Med Hyg 2000;94(4):395-398. https://doi.org/10.1016/s0035-9203(00)90117-2

7. Sandgren A, Hollo V, van der Werf MJ. Extrapulmonary tuberculosis in the European Union and European Economic Area, 2002 to 2011. Euro Surveill 2013;18(12)pii:20431. https://www. eurosurveillance.org/content/10.2807/ese.18.12.20431-en (accessed 2 July 2019).

8. Rock RB, Sutherland WM, Baker C, Williams DN. Extrapulmonary tuberculosis among Somalis in Minnesota. Emerg Infect Dis 2006;12(9):1434-1436. https://doi.org/10.3201/eid1209.050295

9. Sharma SK, Mohan A. Extrapulmonary tuberculosis. Indian J Med Res 2004;120(4):316-353.

10. Harries AD, Hargreaves NJ, Kwanjana JH, Salaniponi FM. The diagnosis of extrapulmonary tuberculosis in Malawi. Trop Doct 2003;33(1):7-11. https://doi.org/10.1177/004947550303300106

11. Harries AD, Maher D, Nunn P. An approach to the problems of diagnosing and treating adult smearnegative pulmonary tuberculosis in high-HIV-prevalence settings in sub-Saharan Africa. Bull World Health Organ 1998;76(6):651-662.

12. Heller T, Goblirsch S, Bahlas S, et al. Diagnostic value of FASH ultrasound and chest X-ray in HIVco-infected patients with abdominal tuberculosis. Int J Tuberc Lung Dis 2013;17(3):342-344. https:// doi.org/10.5588/ijtld.12.0679

13. Corstiens PL, Tjon Kon Fat EM, de Dood CJ, et al. Multi-center evaluation of a user-friendly lateral flow assay to determine IP-10 and CCL4 levels in blood of TB and non-TB cases in Africa. Clin Biochem 2016;49(1-2):22-31. https://doi.org/10.1016/j.clinbiochem.2015.08.013

14. World Health Organization. Automated Real-Time Nucleic Acid Amplification Technology for Rapid and Simultaneous Detection of Tuberculosis and Rifampicin Resistance: Xpert MTB/RIF System: Polic Statement. Geneva: WHO, 2011. http://www.who.int/tb/publications/tb-amplificationtechnology statement/en/ (accessed 17 December 2016)

15. Steingart KR, Sohn H, Schiller I, et al. Xpert(R) MTB/RIF assay for pulmonary tuberculosis and rifampicin resistance in adults. Cochrane Database Syst Rev 2013, Issue 1. Art. No.: CD009593. https:// doi.org/10.1002/14651858.cd009593.pub2

16. Heller T, Wallrauch C, Goblirsch S, Brunetti E. Focused assessment with sonography for HIVassociated tuberculosis (FASH): A short protocol and a pictorial review. Crit Ultrasound J 2012;4(1):21. https://doi.org/10.1186/2036-7902-4-21

17. Brindle HE, Allain TJ, Kampondeni S, et al. Utilization of ultrasound in medical inpatients in Malawi. Trans R Soc Trop Med Hyg 2013;107(7):405-410. https://doi.org/10.1093/trstmh/trt034

18. Groen RS, Leow JJ, Sadasivam V, Kushner AL. Review: Indications for ultrasound use in low- and middle-income countries. Trop Med Int Health 2011;16(12):1525-1535. https://doi.org/10.1111/ j.1365-3156.2011.02868.x

19. Van Hoving DJ, Lamprecht HH, Stander M, et al. Adequacy of the emergency point-of-care ultrasound core curriculum for the local burden of disease in South Africa. Emerg Med J 2013;30(4):312-315. https://doi.org/10.1136/emermed-2012-201358

20. Cegielski JP, Lwakatare J, Dukes CS, et al. Tuberculous pericarditis in Tanzanian patients with and withou HIV infection. Tuber Lung Dis 1994;75(6):429-434. https://doi.org/10.1016/0962-8479(94)90116-3

21. Goblirsch S, Bahlas S, Ahmed M, Brunetti E, Wallrauch C, Heller T. Ultrasound findings in case of extrapulmonary TB in patients with HIV infection in Jeddah, Saudi Arabia. Asian Pac J Trop Di 2014;4(1):14-17. https://doi.org/10.1016/s2222-1808(14)60306-6

22. Pereira JM, Madureira AJ, Vieira A, Ramos I. Abdominal tuberculosis: Imaging features. Eur J Radiol 2005;55(2):173-180. https://doi.org/10.1016/j.ejrad.2005.04.015

23. Sculier D, Vannarith C, Pe R, et al. Performance of abdominal ultrasound for diagnosis of tuberculosis in HIV-infected persons living in Cambodia. J Acquir Immune Defic Syndr 2010;55(4):500-502. https://doi.org/10.1097/qai.0b013e3181e6a703

24. Sinkala E, Gray S, Zulu I, et al. Clinical and ultrasonographic features of abdominal tuberculosis in HIV positive adults in Zambia. BMC Infect Dis 2009:9:44. https://doi.org/10.1186/1471-2334-9-44

25. Brunetti EL, Brigada RL, Poletti FL, et al. Die Rolle der Abdomen-Sonographie in der klinischen Betreuung von Aids-Patienten. Ultraschall Med 2006;27(1):20-33. https://doi. klinischen Betreuung vo
org/10.1055/s-2005-858649

26. Dos Santos RP, Scheid KL, Willers DMC, Goldani LZ. Comparative radiological features of disseminated disease due to Mycobacterium tuberculosis vs non-tuberculosis mycobacteria among AIDS patients in Brazil. BMC Infect Dis 2008;8:24. https://doi.org/10.1186/1471-2334-8-24

27. Giordani MT, Brunetti E, Binazzi R, et al. Extrapulmonary mycobacterial infections in a cohort of HIV-positive patients: Ultrasound experience from Vicenza, Italy. Infection 2013;41(2):409-414 https://doi.org/10.1007/s15010-012-0336-4

28. Heller T, Goblirsch S, Wallrauch C, Lessells R, Brunetti E. Abdominal tuberculosis: Sonographic diagnosis and treatment response in HIV-positive adults in rural South Africa. Int J Infect Dis 2010;14(Suppl 3):e108-e112. https://doi.org/10.1016/j.ijid.2009.11.030

29. World Health Organization. Improving the Diagnosis and Treatment Among Adults and Adolescents: Recommendations for HIV-prevalent and Resource-constrained Settings. Geneva: WHO, 2007. http:// Recommendations for HIV-prevalent and Resource-constrained Settings

30. Agarwal D, Narayan S, Chakravarty J, Sundar S. Ultrasonography for diagnosis of abdominal tuberculosis in HIV infected people. Indian J Med Res 2010;132:77-80.

31. Tarantino L, Giorgio A, de Stefano G, et al. [Diagnosis of disseminated mycobacterial infection in AIDS patients by US-guided fine needle aspiration biopsy of lymphnodes and spleen]. Infez Med 2004;12(2):27-33. https://doi.org/10.1007/s00261-003-0035-9

32. Sutherland T, Temple F, Hennessy O, Lee WK. Abdomen's forgotten organ: Sonography and CT of focal splenic lesions. J Med Imaging Radiat Oncol 2010;54(2):120-128. https://doi.org/10.1111/j.1754 9485.2010.02149.

33. Shamseer L, Moher D, Clarke M, et al. Preferred reporting items for systematic review and metaanalysis protocols (PRISMA-P) 2015: Elaboration and explanation. BMJ 2015;349:g7647. https://do org/10.1136/bmj.g7647

34. Whiting PF, Rutjes AW, Westwood ME, et al. QUADAS-2: A revised tool for the quality assessment of diagnostic accuracy studies. Ann Intern Med 2011;155(8):529-536. https://doi.org/10.7326/00034819-155-8-201110180-00009

35. Freeman MF, Tukey JW. Transformations related to the angular and the square root. Ann Math Stat 1950;21(4):607-611. https://doi.org/10.1214/aoms/1177729756

36. Dersimonian R, Laird N. Meta-analysis in clinical trials. Control Clin Trials 1986;7(3):177-188. https:// doi.org/10.1016/0197-2456(86)90046-2

37. Higgins JP, Thompson SG. Quantifying heterogeneity in a meta-analysis. Stat Med 2002;21(11):15391558. https://doi.org/10.1002/sim.1186

38. Egger M, Davey Smith G, Schneider M, Minder C. Bias in meta-analysis detected by a simple, graphical test. BMJ 1997;315(7109):629-634. https://doi.org/10.1136/bmj.315.7109.629

39. Murray JG, Patel MD, Lee S, Sandhu JS, Feldstein VA. Microabscesses of the liver and spleen in AIDS: Detection with 5-MHz sonography. Radiology 1995;197(3):723-727. https://doi.org/10.1148 radiology.197.3.7480745

40. Porcel-Martin A, Rendon-Unceta P, Bascunana-Quirell A, et al. Focal splenic lesions in patient with AIDS: Sonographic findings. Abdom Imaging 1998;23(2):196-200. https://doi.org/10.1007/ s002619900322

41. Méndez N, di Lonardo M, Sawicki M, Gancedo E, Rasgido A, Fainboim H. La ultrasonografí abdominal como método diagnóstico orientador en tuberculosis y/o micobacteriosis en pacientes H.I.V. Rev Argent Radiol 1995;59(2):87-91.
42. Patel MN, Beningfield S, Burch V. Abdominal and pericardial ultrasound in suspected extrapulmonary disseminated tuberculosis. S Afr Med J 2011:101(1):39-42. https://doi.org/10.7196/SAMJ.4201

43. Dominguez-Castellano A, Yanez P, Muniain MA, et al. [The usefulness of abdominal echography in the diagnosis of extrapulmonary tuberculosis in patients with HIV infection]. Enferm Infecc Microbiol Clin 1998;16(2):61-65.

44. Monill-Serra JM, Martinez-Noguera A, Montserrat E, Maideu J, Sabate JM. Abdominal ultrasound findings of disseminated tuberculosis in AIDS. J Clin Ultrasound 1997;25(1):1-6. https://doi. org/10.1002/(sici) 1097-0096(199701)25:1<1::aid-jcul >3.0.co;2-m

45. Akinkuolie AA, Adisa AO, Agbakwuru EA, Egharevba PA, Adesunkanmi AR. Abdominal tuberculosis in a Nigerian teaching hospital. Afr J Med Med Sci 2008;37(3):225-229.

46. Garcia de Casasola Sanchez G, Torres Macho J, Casas Rojo JM, et al. Abdominal ultrasound and medical education. Rev Clin Esp 2014;214(3):131-136. https://doi.org/10.1016/j.rce.2013.09.006

47. Garcia-Casasola G, Sanchez FJ, Luordo D, et al. Basic abdominal point-of-care ultrasound training in the undergraduate: Students as mentors. J Ultrasound Med 2016;35(11):2483-2489. https://doi. org/10.7863/ultra.15.11068

48. Aubry P, Reynaud JP, Nbonyingingo C, Ndabaneze E, Mucikere E. [Ultrasonographic data of the solid organs of the abdomen in stage IV human immunodeficiency virus infection. A prospective study of 101 cases in central Africa]. Ann Gastroenterol Hepatol (Paris) 1994;30(2):43-52.

49. Riera M, Altes J, Homar F, et al. [Fever of unknown origin in patients with HIV infection]. Enferm Infecc Microbiol Clin 1996;14(10):581-585.

50. O'Keefe EA, Wood R, van Zyl A, Cariem AK. Human immunodeficiency virus-related abdominal pain in South Africa: Aetiology, diagnosis and survival. Scand J Gastroenterol 1998;33(2):212-217. https:// doi.org/10.1080/00365529850166978

51. Bernabeu-Wittel M, Villanueva JL, Pachón J, et al. Etiology, clinical features and outcome of splenic microabscesses in HIV-infected patients with prolonged fever. Eur J Clin Microbiol Infect Dis 1999;18(5):324-329. https://doi.org/10.1007/pl00015013

52. Schinina V, Rizzi EB, Mazzuoli G, David V, Bibbolino C. US and CT findings in splenic focal lesions in AIDS. Acta Radiol 2000;41(6):616-620. https://doi.org/10.1080/028418500127346018

53. Barthwal MS, Rajan KE, Deoskar RB, Sharma SK. Extrapulmonary tuberculosis in human immunodeficiency virus infection. Med J Armed Forces India 2005;61(4):340-341. https://doi. org/10.1016/s0377-1237(05)80059-0

54. Maniar JK, Kamath RR, Mandalia S, Shah K, Maniar A. HIV and tuberculosis: Partners in crime. Indian J Dermatol Venereol Leprol 2006;72(4):276-282. https://doi.org/10.4103/0378-6323.26723

55. Clarke DL, Thomson SR, Bissetty T, Madiba TE, Buccimazza I, Anderson F. A single surgical unit's experience with abdominal tuberculosis in the HIV/AIDS era. World J Surg 2007;31(5):1087-1096; discussion 1097-1098. https://doi.org/10.1007/s00268-007-0402-8

56. Chakraborty PP, Bandyopadhyay D. Utility of abdominal ultrasonography in HIV patients. Singapore Med J 2009;50(7):710-714

57. Ghiya R, Naik E, Casanas B, Izurieta R, Marfatia Y. Clinico-epidemiological profile of HIV/TB coinfected patients in Vadodara, Gujarat. Indian J Sex Transm Dis 2009;30(1):10-15. https://doi. org $/ 10.4103 / 0253-7184.55472$

8. Bélard S, Heller T, Orie V, et al. Sonographic findings of abdominal tuberculosis in children with pulmonary tuberculosis. Pediatr Infect Dis J 2017;94(1):8-21. https://doi.org/10.1097/ inf. 0000000000001590

59. Heller T, Mtemangombe EA, Huson MA, et al. Ultrasound for patients in a high HIV/tuberculosis prevalence setting: A needs assessment and review of focused applications for sub-Saharan Africa. Int J Infect Dis 2017;56:229-236. https://doi.org/10.1016/j.jiji.2016.11.001

60. Mbanjumucyo G, Henwood PC. Focused assessment with sonography for HIV-associated tuberculosis (FASH) case series from a Rwandan district hospital. Afr J Emerg Med 2016;6(4):198-201. https://doi. org/10.1016/j.afiem.2016.07.001

61. Mntonintshi M, O'Mahony D Mabunda S, Namugenyi KAF. Undiagnosed tuberculosis in patients with HIV infection who present with severe anaemia at a district hospital. Afr J Prim Health Care Fam Med 2017;9(1):e1-e6. https://doi.org/10.4102/phcfm.v9i1.1406

62. Spalgais S, Agarwal U, Sarin R, Chauhan D, Yadav A, Jaiswal A. Role of routine abdominal ultrasonography in intensified tuberculosis case finding algorithms at HIV clinics in high TB burden settings. BMC Infect Dis 2017;17:351. https://doi.org/10.1186/s12879-017-2433-6

63. Weber SF, Saravu K, Heller T, et al. Point-of-care ultrasound for extrapulmonary tuberculosis in India: A prospective cohort study in HIV-positive and HIV-negative presumptive tuberculosis patients. Am J Trop Med Hyg 2017;98(1):266-273 https://doi.org/10.4269/ajtmh.17-0486

64. Franzen SRP, Chandler C, Siribaddana S, Atashili J, Angus B, Lang T. Strategies for developing sustainable health research capacity in low and middle-income countries: A prospective, qualitative study investigating the barriers and enablers to locally led clinical trial conduct in Ethiopia, Cameroon and Sri Lanka. BMJ Open 2017;7(10):e017246. https://doi.org/10.1136/bmjopen-2017-017246

65. Shroff ZC, Javadi D, Gilson L, Kang R, Ghaffar A. Institutional capacity to generate and use evidence in LMICs: Current state and opportunities for HPSR. Health Res Policy Syst 2017;15:94. https://doi. org/10.1186/s12961-017-0261-1

66. Oliver S, Bangpan M, Stansfield C, Stewart R. Capacity for conducting systematic reviews in lowand middle-income countries: A rapid appraisal. Health Res Policy Syst 2015;13:23. https://doi. org/10.1186/s12961-015-0012-0

Accepted 21 January 2019.

\section{Appendix 1. Database search terms PubMed}

("Ultrasonography"[mesh:noexp] OR "Echocardiography"[Mesh] OR "Ultrasonography, Doppler"[mesh] OR "Tuberculosis/ ultrasonography"[Mesh] OR ultrasound[tiab] OR ultrasonograph ${ }^{\star}[$ tiab] OR ulrasonic[tiab] OR echocardiog*[tiab] OR songraph ${ }^{\star}[$ tiab] OR fash[tiab]) AND (“Tuberculosis”[Mesh] OR tuberculosis[tiab] OR tubercular[tiab])

\section{Embase}

('echography'/exp/mj OR ultrasound:ab,ti OR ultrasonograph*:ab,ti OR sonograph*:ab,ti OR ultrasonic:ab,ti OR echocardiog*:ab,ti 
OR fash:ab,ti) AND ('tuberculosis'/exp OR tuberculosis:ab,ti OR tubercular:ab,ti)

echography/exp was too sensitive and returned well over 4000 records. Limited to major topic. [note carried from original notes]

\section{Web of Science}

Indexes=SCI-EXPANDED, CPCI-S Timespan $=$ All years

TS $=\left(\left(\right.\right.$ ultrasound OR ultrasonograph ${ }^{\star}$ OR sonograph ${ }^{\star}$ OR ultrasonic OR echocardiog ${ }^{\star}$ OR fash) AND (tuberculosis OR tubercular))

\section{CINAHL}

(MH ("Ultrasonography" OR "Echocardiography" OR "Ultrasonography, Doppler") OR TI (ultrasound OR ultrasonograph* OR sonograph* OR ultrasonic OR echocardiog* OR fash) OR AB (ultrasound OR ultrasonograph ${ }^{\star}$ OR sonograph ${ }^{\star}$ OR ultrasonic OR echocardiog* OR fash))
AND

((MH “Tuberculosis+") OR TI (tuberculosis OR tubercular) OR AB (tuberculosis OR tubercular))

\section{LILACS}

(ultrasound OR ultrasonography OR sonography OR ultrason OR echocardiogaphy OR fash OR ecografia OR ultrasonografia OR ecocardiógrafia OR sonografia) AND (tuberculosis OR tubercular OR tuberculose OR tuberculeuse)

\section{AIM}

(ultrasound OR ultrasonography OR sonography OR ultrason OR echocardiogaphy OR fash OR ecografia OR ultrasonografia OR ecocardiógrafia OR sonografia) AND (tuberculosis OR tubercular OR tuberculose OR tuberculeuse) 